\title{
Enfances, Familles, Générations
}

\section{Représentations sociales et parentalité : les points de vue des parents présentant une déficience ou une lenteur intellectuelle}

\section{Élise Milot, Daniel Turcotte et Sylvie Tétreault}

Numéro 22, printemps 2015

URI : https://id.erudit.org/iderudit/1031124ar

DOI : https://doi.org/10.7202/1031124ar

Aller au sommaire du numéro

Éditeur(s)

INRS-UCS

ISSN

1708-6310 (numérique)

Découvrir la revue

Citer cet article

Milot, É., Turcotte, D. \& Tétreault, S. (2015). Représentations sociales et parentalité : les points de vue des parents présentant une déficience ou une lenteur intellectuelle. Enfances, Familles, Générations, (22), 182-205. https://doi.org/10.7202/1031124ar
Résumé de l'article

Cet article présente les résultats d'une étude exploratoire québécoise portant sur les représentations sociales (RS) de la parentalité des personnes ayant une déficience ou une lenteur intellectuelle (DI/LI). Neuf parents ont participé à des entrevues individuelles semi-structurées. Pour les cinq répondants ayant conservé la garde de leurs enfants, la parentalité symbolise l'accès à un rôle hautement désiré dont l'exercice suscite fierté, valorisation, reconnaissance sociale et détermination. Pour ceux qui vivent leur parentalité à travers l'expérience du placement de leurs enfants, leur représentation est davantage teintée par des sentiments d'injustice, de souffrance et de disqualification. Ceux-ci émergent en effet de leurs échanges avec les intervenants en protection de la jeunesse et les membres de leur entourage. La réflexion proposée porte sur les facteurs pouvant freiner ou favoriser le développement d'une identité parentale positive et un réseau de soutien à la parentalité adéquat. 


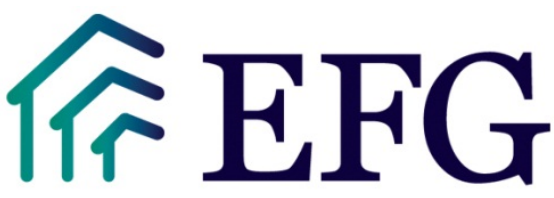

No. 22, 2015, p. 182-205

efg.inrs.ca

REVUE INTERNATIONALE

ENFANCES FAMILLES GÉNÉRATIONS

\title{
Représentations sociales et parentalité : les points de vue des parents présentant une déficience ou une lenteur intellectuelle
}

\author{
Élise Milot \\ Doctorante, École de service social \\ Université Laval (Canada) \\ elise.milot.1@ulaval.ca

\section{Daniel Turcotte} \\ Professeur titulaire, École de service social \\ Université Laval (Canada) \\ daniel.turcotte@svs.ulaval.ca

\section{Sylvie Tétreault} \\ Professeure titulaire, Département de réadaptation \\ Université Laval (Canada) \\ sylvie.tetreault@rea.ulaval.ca
}

\section{Résumé}

Cet article présente les résultats d'une étude exploratoire québécoise portant sur les représentations sociales $(R S)$ de la parentalité des personnes ayant une déficience ou une lenteur intellectuelle (DI/LI). Neuf parents ont participé à des entrevues individuelles semi-structurées. Pour les cinq répondants ayant conservé la garde de leurs enfants, la parentalité symbolise l'accès à un rôle hautement désiré dont l'exercice suscite fierté, valorisation, reconnaissance sociale et détermination. Pour ceux qui vivent leur parentalité à travers l'expérience du placement de leurs enfants, leur représentation est davantage teintée par des sentiments d'injustice, de souffrance et de disqualification. Ceux-ci émergent en effet de leurs échanges avec les intervenants en protection de la jeunesse et les membres de leur entourage. La réflexion proposée porte sur les facteurs pouvant freiner ou favoriser le développement d'une identité parentale positive et un réseau de soutien à la parentalité adéquat.

Mots clés : Représentations sociales, attitudes, parentalité, soutien à la famille, identité parentale 


\begin{abstract}
The present article describes the results of a Quebec exploratory study dealing with the social representations (SR) of parenthood experienced by people with intellectual disabilities or cognitive difficulties (ID/CD). Nine parents took part in individual semistandardized interviews. As regards the five interviewees who have maintained guardianship of their children, parenthood symbolizes access to an extremely desirable role, the exercise of which stimulates pride, personal uplift, social recognition and commitment. For those whose parenthood is subject to the foster placement of their children, their arguments are blemished due to their feelings of injustice, of suffering and of demotion. These feelings are indeed made clear in their discussions with the child protection representatives and members of their families. The ideas proposed deal with factors likely to slow down or to encourage the development of a positive parental identity and a sufficient parenthood support network.
\end{abstract}

Keywords: Social representation, attitudes, parenthood, family support, parental identity

Aujourd'hui, devenir parent s'avère un choix convoité par un nombre grandissant d'adultes vivant avec une déficience ou une lenteur intellectuelle (DI/LI) ${ }^{1}$. Il s'agit d'une décision faisant l'objet d'un droit légalement reconnu (Llewellyn et al., 2010). Toutefois, la croyance voulant qu'il soit impossible pour ces personnes d'assumer la responsabilité d'un enfant demeure prégnante (Aunos et Feldman, 2002; Coppin, 2004; Llewellyn et McConnell, 2010a). Or, un tel positionnement est susceptible d'avoir des incidences importantes. En effet, il conditionne les attitudes et les comportements à l'endroit des personnes présentant une DI/LI qui choisissent de devenir parents et influence, par le fait même, la construction de leur identité parentale. C'est là où se situe la proposition sous-jacente à cette recherche qui s'intéresse au regard porté par des parents ayant une DI/LI sur cette parentalité singulière. Le texte se divise en cinq sections, soit : 1) la problématique et les objectifs de recherche; 2) la méthodologie; 3) les résultats; 4) la discussion; 5) les limites et les considérations méthodologiques.

\title{
1. Problématique
}

Selon Lamour et Barraco (1998), la parentalité se définit comme l'ensemble du processus permettant à des adultes d'exercer leur rôle parental, soit de répondre aux besoins de leurs enfants sur le plan physique, affectif et psychologique. Au-delà du rôle, la parentalité peut également être abordée sous l'angle de l'identité. Composante de la parentalité, l'identité parentale renvoie à la manière dont chacun se définit, s'estime et se présente en tant que parent, à autrui et à soi-même (Euillet et Zaouche-Gaudron, 2008). Elle est tributaire de l'expérience subjective et modulée par l'interaction de variables personnelles et contextuelles (Robin et Fontaine, 2013). Certaines expériences peuvent avoir une influence majeure sur l'identité parentale, telles que la séparation du parent et de son enfant lorsqu'il y a retrait de la garde par les autorités légales. Cette situation a des répercussions directes non seulement sur l'identité parentale, mais également sur leur estime de soi (Euillet et Zaouche-Gaudron, 2008).

\footnotetext{
${ }^{1}$ Cette étude emploie le terme DI/LI qui fait référence aux personnes ayant un diagnostic établi où présentant des déficits sur le plan cognitif et adaptatif caractéristiques d'une lenteur intellectuelle, aux limites du diagnostic.
}

É. Milot, D. Turcotte et S. Tétreault, Représentations sociales et parentalité : les points de vue... Enfances Familles Générations, no 22, 2015, p. 182-205 - www.efg.inrs.ca 
En plus de susciter une double disqualification, à la fois éducative et sociale, elle cause des sentiments de dépréciation de soi et de souffrance (Sécher, 2010; Sellenet, 2000).

Indéniablement, être parent représente une dimension de la vie à la fois gratifiante et source de défis. Pour les parents présentant une DI/LI, les défis s'en trouvent accrus lorsque sont considérées certaines variables personnelles et contextuelles (MirfinVeitch, 2010). Or, pour avoir une vision globale de la situation, il faut aussi s'attarder aux ressources sur lesquelles ils peuvent compter.

\subsection{Variables individuelles}

L'examen des défis que pose l'exercice de la parentalité chez les personnes présentant une DI/LI ne peut se faire sans s'attarder aux répercussions des caractéristiques personnelles, notamment leur histoire et leurs limitations cognitives et adaptatives.

Plusieurs personnes vivant avec une DI/LI ont une histoire de vie marquée par la stigmatisation et la rupture répétée des liens affectifs (Coppin, 2007; Llewellyn et McConnell, 2010b). Dans certains cas, elles n'ont pas pu compter sur des modèles parentaux adéquats, parce qu'elles vivaient en centre d'accueil ou que leur vie familiale était chaotique et dysfonctionnelle. Dans d'autres cas, elles ont été victimes de mauvais traitements pendant leur enfance. Ces facteurs peuvent altérer leur capacité à construire des relations de proximité positives (Feldman, 2002). Coppin (2004) constate que peu de parents présentant une DI/LI ont bénéficié de la préparation nécessaire à un choix éclairé de parentalité. Alors que certains ont été peu ou pas conscientisés aux responsabilités sous-jacentes à ce rôle (McGraw et Candy, 2010), d'autres n'ont jamais été mis en situations concrètes de pratique (p. ex. gardiennage) (Coppin, 2003). Ainsi, ces parents n'ont jamais été amenés à réfléchir sur l'ampleur des responsabilités et sur les ressources dont ils disposent avant de concrétiser leur désir d'enfant (Ledain, 2008).

Les aptitudes parentales sont aussi tributaires des capacités cognitives et des habiletés liées à la réalisation des activités de la vie quotidienne (Aunos et Feldman, 2010). Plusieurs difficultés transversales peuvent influencer directement la manière dont les parents présentant une DI/LI exercent leur rôle parental, par exemple se projeter dans le temps ou utiliser le langage pour exprimer sa pensée (Coppin, 2004). L'absence ou l'inefficacité des savoir-faire ou des savoir-être complexifie l'exercice de leur parentalité. Le déficit cognitif n'est cependant pas le seul prédicteur de leur succès parental. Celui-ci est tout autant tributaire de variables pouvant caractériser leur vécu, telles que la pauvreté, l'isolement social, un réseau limité de ressources de soutien (Aunos et Feldman, 2010; Sigurjónsdóttir et Traustadóttir, 2010) ou un haut niveau de stress parental (Booth et Booth, 1998; Pixa-Kettner, 1999; Rao, 2013).

\subsection{Variables contextuelles}

Selon Preston (2012), les messages qui sont actuellement véhiculés dans les sociétés contemporaines traduisent, à la fois de façon implicite et explicite, que la sexualité, les relations de couple et le « devenir parent » ne sont pas des options envisageables pour les adultes présentant une DI/LI. Être parent exige le développement de compétences associées à la maturité et au sens des responsabilités (Poussin, 2004), des concepts opposés à l'image de l'enfant simple d'esprit souvent accolée à l'adulte présentant une DI (Llewellyn et McConnell, 2010a). L'idée voulant que tout adulte présentant ce profil devienne inévitablement un parent déficient circule encore (Coppin, 2007). Ces messages invalidants, découlant d'une vision stéréotypée de la DI, sont plus ou moins intériorisés par les personnes présentant ce profil (Chatroussat, 2011; Coppin, 2001; 
Leroux et Scelles, 2007; Pasleau et Collignon, 1997). Conséquemment, l'accès au rôle de parent s'en trouve plus difficile à concevoir.

Or une telle représentation limite l'accès à une expérience enrichissante. En effet, devenir parent s'accompagne non seulement d'un nouveau statut, mais ouvre à des relations et à des responsabilités nouvelles (Booth et Booth, 2005). L'exercice de la parentalité permet aux personnes présentant une DI/LI d'acquérir une identité aucunement liée à leur condition. Il justifie la fréquentation de milieux normalisants, tels que l'école, ce qui peut alimenter leur impression de mener une vie similaire à celle des autres parents (Booth et Booth, 1995). Le statut de parent renforce le sentiment d'appartenance, de réalisation et d'accomplissement (Llewellyn et Gustavsson, 2010). Il symbolise l'engagement personnel à un futur que ces personnes se créent pour ellesmêmes, lequel se trouve parfois en opposition avec ce que leurs proches souhaiteraient (McConnell, 2008).

\subsection{Ressources}

De nombreuses personnes présentant une DI/LI peuvent être ou devenir de bons parents lorsqu'elles bénéficient de services adaptés à leurs besoins (Feldman, 2002, 2010; Wade et al., 2007). Par contre, une coopération efficiente entre les parents et les professionnels impliqués est nécessaire (Feldman, 1994; Pixa-Kettner, 2008). Pourtant, celle-ci se bute à différents obstacles. D'une part, les pratiques sont parfois limitées par la présence de préjugés. En effet, les difficultés des parents sont souvent perçues comme insurmontables, permanentes (Booth et Booth, 2004; Gray, 2011; Swain et Cameron, 2003) et rendant impossible l'acquisition des compétences nécessaires à une réponse adaptée aux besoins évolutifs d'un enfant (McConnell, 2008; McConnell et Sigurjónsdóttir, 2010; Tarleton, 2013). D’autant plus que les intervenants sociaux se sentent généralement démunis face à ces personnes, invoquant leurs connaissances et une expertise limitées dans l'intervention auprès de cette clientèle, l'absence de temps pour se perfectionner, une collaboration problématique avec les partenaires et un délai insuffisant pour réaliser l'intervention (Booth et al., 2006).

D'autre part, les parents se sentent souvent mal à l'aise dans leurs interactions avec les intervenants sociaux (Gray, 2011). Un sentiment d'incompréhension est évoqué à l'égard de la fonction des services proposés et du rôle des différents professionnels (Booth et al., 2006; Painz, 1993; Tymchuk et al., 1999). Leurs visites sont parfois vécues par les parents comme une intrusion à la vie privée, car ils s'y sentent plus jugés qu'aidés (Booth et Booth, 1995). Ils éprouvent un sentiment d'être toujours surveillés par les intervenants, et parfois, par des membres du voisinage et de leur famille. Cette impression nuit à leur capacité à assumer pleinement leurs responsabilités parentales (Leroux et Scelles, 2007). Perçue tel un manque de confiance en leurs capacités, cette surveillance amplifie parfois leur sentiment de ne pas être à la hauteur. Conséquemment, plusieurs parents éprouvent de la crainte et de la résistance à l'idée de demander de l'aide, ou encore ils refusent d'accepter les services offerts (Traustadóttir et Sigurjónsdóttir, 2010). Somme toute, cette sensation d'être perçus de manière négative perturbe la relation parents-intervenants et freine la mise en place de mécanismes efficaces de soutien à la parentalité.

Selon des études consultées, la source de soutien privilégiée par les parents présentant une DI/LI provient des membres de la famille élargie (Ehlers-Flint, 2002; Guinea, 2001). Selon Llewellyn et McConnell (2002), plusieurs mères présentant une DI/LI se sentent plus à l'aise de recevoir de l'aide ou des conseils de leurs proches plutôt que des professionnels. Ce type de soutien est encore mieux accueilli lorsque son actualisation rehausse leur confiance en leurs compétences parentales et s'arrime à leurs attentes et à leurs valeurs (Llewellyn, 1995). Or plusieurs parents présentant une 
DI/LI n'ont aucun ou très peu de contacts avec leur famille élargie (Llewellyn et McConnell, 2002). D'autres n'apprécient pas l'attitude de leurs proches lorsqu'ils ont des comportements semblant témoigner d'une disqualification de leurs pratiques et de leurs compétences parentales (Mayes et al., 2008; McConnell et Sigurjónsdóttir, 2010).

Si plusieurs facteurs exercent une influence sur l'exercice de la parentalité, il ne faut pas négliger le regard que portent les parents sur leur propre situation. À cet égard, l'examen de leurs représentations sociales de la parentalité chez les personnes vivant avec une DI/LI offre une avenue pertinente et originale pour développer une meilleure compréhension de leur réalité.

\section{4 À propos des représentations sociales}

\subsubsection{Définition}

Une représentation sociale (RS) renvoie à un ensemble organisé d'opinions, d'attitudes, de croyances et d'informations se référant à un objet ou à une situation, qui est déterminé à la fois par l'individu lui-même, par le système social et idéologique dans lequel il est inséré et par la nature des liens qu'il entretient avec ce système social (Abric, 2009). Cette interprétation de la réalité permet de donner un sens à ses conduites et de comprendre la réalité à travers son propre système de référence (Rateau, 2007). Deux composantes définissent la RS : ses éléments constitutifs et son organisation, c'est-à-dire les relations qu'entretiennent ces éléments entre eux (Jodelet, 2009). Selon Moliner, Rateau et Cohen-Scali (2002 : 12), ces éléments constitutifs d'une RS peuvent indifféremment être qualifiés d'opinions, d'informations et de croyances, car « la frontière est souvent floue entre le je pense, le je sais et le je crois ». Il s'agit donc de retenir qu'une telle représentation se présente concrètement comme un ensemble d'éléments cognitifs et sociocognitifs relatifs à un objet social.

\subsubsection{Dimensions du système représentationnel}

Selon Moscovici (1961), le système représentationnel s'élabore autour de trois dimensions. La première fait référence à l'ensemble d'informations ou à la somme des connaissances des personnes à propos de l'objet social. Ces connaissances peuvent être plus ou moins variées, originales ou stéréotypées (Herzlich, 1972). Pour Jodelet (2012), l'ensemble de ces connaissances a une visée pratique, puisqu'elles influencent les comportements des individus vis-à-vis d'un phénomène ou d'un évènement qui se présente à eux. La deuxième dimension est l'attitude générale qui marque les dispositions favorables ou non d'une personne à l'égard de l'objet de représentation. L'attitude a une valence (positive ou négative) et varie en intensité (Vallerand, 2006). Cette orientation détermine la sélection des informations et leur hiérarchisation dans le champ. Enfin, la troisième dimension est le champ de représentation et se définit telle une structure intériorisée qui emmagasine, organise, hiérarchise et articule les différents éléments d'information à l'égard d'un objet. Cette organisation structurante des connaissances connues permet à la personne de se constituer une image évocatrice de l'objet de représentation. Ainsi, ce champ de représentation, qui est variable d'un individu à l'autre, permet de décoder les informations ultérieures et de préparer, du même coup, des comportements adaptés à son environnement social (Herzlich, 1972; Pasleau et Collignon, 1997). En plus de ces dimensions, il importe de considérer le contexte dans lequel s'inscrit la représentation. Celui-ci fait référence non seulement à l'environnement immédiat où évolue le groupe social, mais aussi aux valeurs et idéologies communes à l'ensemble d'une société auxquelles se réfèrent ses membres afin de se représenter un objet (Abric, 1994). 
L'étude présentée dans cet article vise principalement l'exploration des composantes du système représentationnel des participants, lesquelles proviennent des propos des sujets amenés à se prononcer sur l'objet étudié (Abric, 2003). Le rapport à l'exercice de la parentalité a été choisi comme angle d'analyse, tenant pour acquis que les participants vivaient dans des conditions similaires au moment de l'étude.

\subsubsection{Critères de reconnaissance de l'objet de RS}

Afin de s'assurer que l'objet social ciblé dans une démarche de recherche est un objet de représentation sociale, Moliner (1993) propose de considérer différents critères, soit les spécificités de l'objet, les caractéristiques du groupe, les enjeux, la dynamique sociale et l'absence d'orthodoxie. Cette section définit ces critères et spécifie en quoi la parentalité des personnes vivant avec une DI/LI est un objet de RS chez les parents présentant cette condition.

Tout d'abord, ce n'est pas tant la nature de l'objet de représentation qui importe, mais bien son statut social. Selon Moliner (1993), l'objet doit être important pour des individus, il doit représenter une menace ou susciter de l'intérêt. À ce sujet, la parentalité symbolise l'accès à un rôle hautement désiré et à la possibilité de développer une image de soi plus positive pour les personnes présentant une DI/LI (Edmonds, 2000; Mayes et al., 2011). Conséquemment, devenir parent s'avère le choix convoité par un nombre grandissant d'adultes vivant avec une DI/LI. De plus, l'objet de représentation doit être polymorphe. Ceci signifie qu'un même objet peut revêtir plusieurs formes à divers moments au sein de groupes différents (Mariotti, 2003). Il doit aussi correspondre à une classe d'objets (Moliner, 1993). D'une part, la parentalité des personnes présentant une DI/LI est polymorphe, puisqu'elle s'est présentée sous différentes formes à travers le temps, et ce, pour divers groupes sociaux. Alors qu'il était jadis strictement défendu à ces personnes, l'exercice de cette parentalité fait maintenant l'objet d'un droit reconnu dont l'actualisation se doit d'être soutenue par une offre de services adaptés. Ainsi, l'émergence de cette nouvelle réalité a bousculé les attitudes et les réactions des personnes présentant une DI/LI, de leurs proches et des différents groupes de professionnels offrant des services publics généraux ou spécialisés au cours des dernières décennies (Lalande et al., 2002). D'autre part, cet objet de représentation recouvre plusieurs aspects spécifiques faisant référence aux droits et aux devoirs du parent, aux soins prodigués à l'enfant et à l'expérience de la parentalité, soit le vécu et les sentiments du parent (Houzel, 1999, 2002).

L'étude d'une représentation au sein d'un groupe donné nécessite que l'objet ait une présence thématique récurrente dans les communications (implicite ou explicite) entre les membres du groupe (Flament et Rouquette, 2003; Moliner, 1993). À ce sujet, la parentalité des personnes vivant avec une DI/LI chez les parents présentant ce profil, et qui reçoivent des services professionnels permettant de pallier leurs difficultés, détient une telle saillance dans les propos entretenus au sein de ce groupe.

Si l'objet de représentation est d'une utilité ou d'une valeur sociale pour les membres d'un groupe, c'est parce qu'il est porteur d'enjeux (Moliner et al., 2002). Ces enjeux se dévoilent dans les interactions entre ce groupe et d'autres groupes, ce qui amène Moliner $(1993,1996)$ à en conclure qu'un objet de représentation se retrouve toujours inséré dans une dynamique sociale. Cette dernière est caractérisée par trois composantes indispensables: le groupe social, l'objet et l'autrui social. Ainsi, «la représentation est bien représentation de quelque chose, produite par quelqu'un (ou quelques-uns) », une production qui se fait "par rapport à quelqu'un d'autre » (Moliner, 1993 : 11). Ainsi, par son positionnement dans la société, chaque groupe peut avoir une pratique et une expérience spécifiques de l'objet de représentation (Moliner et al., 2002). Selon ces mêmes auteurs, «les différents groupes sociaux qui sont en 
interaction autour (ou à cause) d'un objet social vont se représenter cet objet selon une certaine logique et conformément à certains intérêts » (p. 18). Considérant les enjeux sociaux et éthiques qu'il soulève, la parentalité des personnes présentant une DI/LI représente une thématique qui apparaît de manière récurrente dans le discours contemporain (Coppin, 2004) et, nécessairement, dans les interactions entre les parents vivant avec une DI/LI et les intervenants sociaux impliqués professionnellement.

Dans un système orthodoxe des pensées, les membres d'un groupe sont assujettis à des instances régulatrices rigides qui fournissent un "prêt-à-penser» de manière à épargner aux membres d'un groupe social toute situation d'incertitude (Deconchy, 2012). Or dans un tel système, une RS ne peut pas émerger, puisque les instances régulatrices contrôlent la diffusion et la validité des informations relatives à l'objet (Moliner, 1993). Ainsi, les parents présentant une DI/LI ne sont pas encadrés par un tel système orthodoxe; ils sont libres d'accéder aux ressources qu'ils désirent, ce qui contribue à forger leur conception particulière de la parentalité des personnes présentant cette condition.

\subsection{Objectifs}

Cette étude porte sur l'exploration des RS de la parentalité des personnes vivant avec une DI/LI chez des parents québécois présentant cette condition. Les objectifs sont: 1) dégager les composantes cognitives (informations) et sociocognitives (opinions, attitudes, croyances) au cœur du système représentationnel; 2) ressortir les convergences et les divergences intragroupes; 3) identifier les facteurs personnels et contextuels liés aux RS.

\section{Méthodologie}

\subsection{Constitution de l'échantillon}

Suivant l'obtention du certificat éthique ${ }^{2}$, le recrutement des participants s'est réalisé principalement par l'entremise d'organismes communautaires. Des exemplaires d'un dépliant d'information adapté ont été offertes aux intervenants des organismes ciblés pour qu'ils sollicitent des participants potentiels. Cette démarche a été guidée par les critères d'inclusion et d'exclusion établis qui sont présentés au tableau 1. Ces critères ont permis la constitution d'un échantillon non probabiliste par choix raisonné (Fortin, 2006). L'objectif était que l'échantillon soit composé de représentants ayant acquis des expériences relativement diversifiées afin d'explorer en profondeur différentes facettes du même phénomène (Rubin et Babbie, 2013). Ce souci visait à favoriser l'induction analytique. Selon Pires (1997 : 151), ce principe consiste à chercher dans un petit nombre de cas concrets, les caractéristiques qui leur sont essentielles (ou les propriétés constitutives), ce qui permet une certaine généralisation, présumant que « parce qu'elles sont essentielles, elles doivent s'appliquer à d'autres cas similaires ».

2 Le devis de cette étude a fait l'objet d'un processus d'évaluation éthique de projet de recherche multicentrique reconnu par le ministère de la Santé et des Services sociaux du Québec (2006). Cette démarche a été approuvée et coordonnée par le Comité d'éthique de la recherche conjoint destiné aux centres de réadaptation en déficience intellectuelle et en troubles envahissants du développement (CÉRC/CRDITED). 
Tableau 1

Critères de l'échantillon

\begin{tabular}{|c|c|}
\hline Critères & \\
\hline $\begin{array}{c}\text { Critères } \\
\text { d'inclusion }\end{array}$ & $\begin{array}{l}\text { - Présenter un diagnostic de DI légère ou bénéficier (ou avoir } \\
\text { bénéficié par le passé) de mesures particulières pour des raisons de } \\
\text { DI soupçonnée (p. ex. avoir fréquenté une classe spéciale, recevoir } \\
\text { un montant de soutien au revenu pour les personnes handicapées, } \\
\text { etc.); } \\
\text { Être parent (peu importe le type de garde); } \\
\text { - } \quad \text { Être âgé de } 21 \text { à } 59 \text { ans; } \\
\text { - } \quad \text { Détenir la capacité de s'exprimer en français de manière autonome; } \\
\text { - } \quad \text { Demeurer dans une des régions du Québec (Canada) suivantes: } \\
\text { Capitale-Nationale, Chaudière-Appalaches, Lanaudière, Mauricie; } \\
\text { - Avoir des contacts avec au moins un intervenant du domaine social. }\end{array}$ \\
\hline $\begin{array}{c}\text { Critères } \\
\text { d'exclusion }\end{array}$ & $\begin{array}{l}\text { - Connaître personnellement ou professionnellement la personne } \\
\text { menant cette recherche (première auteure); } \\
\text { - Présenter un problème majeur de santé mentale; } \\
\text { - Être sous régime de protection (tutelle, curatelle, mandat } \\
\text { homologué). }\end{array}$ \\
\hline
\end{tabular}

\subsection{Collecte de données}

Les données ont été principalement recueillies dans le cadre d'entretiens individuels en face à face. Cette méthode a été privilégiée en raison de son efficacité dans le repérage des contenus des RS (Negura, 2004).

Considérant la situation particulière des répondants, ils ont été rencontrés à trois reprises. D'abord, une rencontre préalable de 45 minutes a été réalisée avec chaque parent à un endroit et à un moment de son choix. Ce premier contact a permis de présenter le formulaire de consentement et d'obtenir leur accord libre et éclairé de participer. Une fiche sociodémographique a aussi été remplie en vue de consigner certains éléments de leur histoire personnelle et de leurs conditions de vie actuelles. Un témoin, choisi par chaque participant et identifié lors du premier échange verbal par téléphone, était également présent lors de cette rencontre. Préalablement à la première entrevue, des informations sur les habiletés de communication des participants ont été recueillies auprès des témoins identifiés par les parents. Cette démarche a permis de personnaliser le guide d'entretien par la référence à un vocabulaire connu ainsi qu'à des personnes, à des activités ou à des lieux de leur quotidien (Julien-Gauthier et al., 2009). Lors des entrevues, cinq participants ont voulu être accompagnés par ce témoin de confiance, et leur souhait a été respecté.

À travers deux entretiens semi-dirigés de 30 minutes, les participants ont répondu à treize différentes questions de ce guide. Il est à souligner que le niveau de complexité grammaticale des questions avait été préalablement adapté après la consultation d'experts (universitaires et intervenants) et un prétest avec un parent présentant une DI. Les questions ont été posées selon le flux conversationnel et les réactions des participants (Nils et Rimé, 2003). Elles visaient l'expression de leur propre expérience à travers différents aspects de leur vie quotidienne. Les questions du guide sont liées aux objectifs et portent sur cinq thèmes : 1) le désir de procréation; 2) l'exercice des droits et des devoirs parentaux; 3) la pratique des tâches et des responsabilités 
parentales; 4) l'expérience de parentalité (vécu subjectif, sentiments); 5) les conditions de vie favorables à l'exercice du rôle parental. Les thèmes liés à chaque question ont été annotés dans ce guide. Les entretiens se sont déroulés entre janvier et mai 2013. Avec le consentement des participants, ils ont été enregistrés par magnétophone, puis retranscrits intégralement.

Toutes les étapes du projet, et plus particulièrement la catégorisation, ont été supervisées par le directeur et la codirectrice de thèse de l'étudiante (première auteure) pour limiter la subjectivité qui peut influencer les inférences réalisées à partir du discours (Mayer et Deslauriers, 2000). Par souci de validité interne, une attention particulière a été portée à l'objectivation de la démarche par la tenue d'un journal de bord, une modalité qui favorise la distanciation du positionnement sur le plan théorique, émotif et social (Laperrière, 1997).

\subsection{Méthode d'analyse des données}

Selon Moliner et al. (2002), l'analyse de contenu est la technique la plus appropriée pour identifier les informations, les opinions, les attitudes et les croyances véhiculées dans les discours. Cette méthode de traitement des communications permet l'accès au sens qui se retrouve derrière les paroles, lequel apparaît a posteriori grâce aux interprétations du chercheur (Bardin, 2003, 2007; Blais et Martineau, 2006). Dans la présente recherche, l'analyse des données a été réalisée en privilégiant une démarche inductive. Ainsi, la démarche, facilitée par le logiciel N'vivo, a suivi trois étapes : 1) la lecture flottante des verbatims; 2) la classification des segments liés aux objectifs d'étude; 3) l'attribution de mots-clés aux sous-catégories émergentes, en respect des principes d'exclusion mutuelle, d'homogénéité et de cohérence (Bardin, 2003, 2007). Les données ont été analysées en distinguant deux sous-groupes de parents: ceux ayant conservé la garde de leurs enfants et ceux dont l'enfant est placé. Finalement, des moyennes et des analyses de fréquence ont été réalisées à partir des données sociodémographiques consignées dans un document Excel.

\section{Résultats}

Cette section renvoie à la description des participants et à la présentation des aspects relatifs aux trois dimensions du système représentationnel pour chaque groupe de parents. À ce sujet, il sera d'abord question de l'image évocatrice de l'objet de représentation, laquelle émerge de l'exploration du champ de représentation. Puis, les éléments relatifs à l'attitude générale et aux connaissances des répondants à l'égard de l'objet d'étude seront présentés.

\subsection{Description des participants}

Tous les volontaires souhaitant participer à cette étude ont été sélectionnés. Toutefois, la contribution d'un des parents n'a pas été retenue, puisque son discours lors des entretiens a semblé fortement influencé par la présence d'un témoin de confiance. Les propos analysés renvoient à ceux de neuf parents, soit six mères et trois pères. Six présentent un diagnostic de DI légère, confirmé par les témoins de confiance. Les trois autres se reconnaissent des limitations cognitives illustrées par les difficultés ayant marqué leur parcours de vie. Celles-ci font référence aux apprentissages scolaires (p. ex. fréquentation de classes spéciales) et à leur intégration sociale (p. ex. mise à pied fréquentes et difficultés à conserver un emploi). Tous Québécois et Québécoises d'origine, ils vivent majoritairement en milieu rural $(n=7)$. Ils sont sans emploi à temps plein et bénéficient de prestations gouvernementales (solidarité sociale). Des informations supplémentaires sont consignées au tableau 2, telles que les ressources significatives de soutien à l'exercice de leur rôle parental. 
Tableau 2

Caractéristiques de l'échantillon ( $\mathrm{n}=9$ )

\begin{tabular}{|c|c|c|c|}
\hline \multicolumn{4}{|c|}{ Parents ayant conservé la garde $(n=5)$} \\
\hline $\begin{array}{c}\text { Nom }^{3} \\
\text { Âge }\end{array}$ & Situation maritale & Situation familiale & Ressources significatives \\
\hline $\begin{array}{l}\text { Fannie } \\
29 \text { ans }\end{array}$ & $\begin{array}{l}\text { Séparée du père, } \\
\text { en couple }\end{array}$ & Vit avec sa fille de 4 ans, dont elle a la garde à temps plein. & $\begin{array}{c}\text { - Intervenant communautaire } \\
\text { - Éducateur des services publics généraux } \\
\text { - Mère et amis } \\
\end{array}$ \\
\hline $\begin{array}{l}\text { Léa } \\
42 \text { ans }\end{array}$ & En couple & Vit avec son conjoint et ses trois enfants de 18,21 et 22 ans. & $\begin{array}{c}\text { - Intervenant communautaire } \\
- \text { Amis }\end{array}$ \\
\hline $\begin{array}{l}\text { Marie } \\
43 \text { ans }\end{array}$ & Célibataire & Vit chez son père avec ses deux fils de 20 et 27 ans. & $\begin{array}{l}\text { - Intervenant communautaire } \\
\text { - Trois sœurs, un frère et amis }\end{array}$ \\
\hline $\begin{array}{l}\text { Alice } \\
48 \text { ans et } \\
\text { Marc } \\
47 \text { ans }\end{array}$ & Mariés & Vivent avec leur fils de 8 ans. & $\begin{array}{c}\text { - Deux éducateurs des services spécialisés (DI) } \\
\text { - Éducateur en répit des services publics généraux } \\
\text { - Gardienne de } 16 \text { ans }\end{array}$ \\
\hline \multicolumn{4}{|c|}{ Parents ayant perdu la garde de l'enfant ou des enfants $(n=4)$} \\
\hline $\begin{array}{c}\text { Vicky } \\
22 \text { ans } \\
\end{array}$ & Célibataire & $\begin{array}{l}\text { Vit chez ses parents. A un fils de } 2 \text { ans placé en centre jeunesse } \\
\text { depuis la naissance. Elle le visite une fois par mois. }\end{array}$ & $\begin{array}{l}\text { - Éducateur des services spécialisés (DI) } \\
\text { - Intervenant communautaire }\end{array}$ \\
\hline $\begin{array}{l}\text { Martin } \\
39 \text { ans }\end{array}$ & Marié & $\begin{array}{l}\text { Vit avec sa conjointe. A une fille de } 12 \text { ans qui vit en famille } \\
\text { d'accueil. II la voit toutes les deux semaines. }\end{array}$ & $\begin{array}{l}\text { - Intervenant communautaire } \\
\text { - Parents, deux frères et amis } \\
\end{array}$ \\
\hline $\begin{array}{l}\text { Philippe } \\
40 \text { ans }\end{array}$ & En couple & $\begin{array}{l}\text { Vit avec sa conjointe et ses enfants. } \\
\text { A un fils de } 15 \text { ans. Il est placé chez les grands-parents maternels } \\
\text { depuis l'âge de } 3 \text { ans. Ils se voient chaque fin de semaine. }\end{array}$ & $\begin{array}{l}\text { - Intervenant communautaire } \\
\text { - Parents, deux frères et amis }\end{array}$ \\
\hline $\begin{array}{l}\text { Caroline } \\
58 \text { ans }\end{array}$ & Célibataire & $\begin{array}{l}\text { Vit seule. A deux filles de } 30 \text { et } 13 \text { ans. Elles ont grandi en familles } \\
\text { d'accueil. Dans le cadre de contacts supervisés, Caroline voit } \\
\text { toujours la plus jeune une fois par mois. }\end{array}$ & $\begin{array}{l}\text { - Travailleur social de la protection de la jeunesse } \\
\text { - Frère et belle-sœur (avant les placements) }\end{array}$ \\
\hline
\end{tabular}

${ }^{3}$ Tous les prénoms ont été changés pour préserver l’anonymat des répondants. 


\subsection{Représentations de la parentalité des parents ayant conservé la garde}

\subsubsection{Image évocatrice de l'objet}

Pour les cinq parents qui ont conservé la garde de leurs enfants, la parentalité suscite valorisation, reconnaissance sociale, fierté et détermination.

Un point commun mentionné par cinq répondants concerne l'accès au statut de parent, c'est-à-dire se sentir reconnu comme étant capable de s'occuper d'un enfant. Cette sensation, qui découle du regard porté par des personnes significatives, témoigne d'une reconnaissance différente et appréciée. Par exemple, trois répondants soulignent avoir été félicités pour leur soutien à la réussite scolaire de l'enfant et pour leur progrès dans la mise en place d'une meilleure discipline. Or assumer l'autorité et la gestion parentales n'est pas toujours une tâche agréable; elle suscite parfois de l'inconfort en raison d'une peur de chagriner son enfant. Comme le spécifie Alice: "C'est difficile, mais quand tu l'appliques, ça va bien après. Au début, j'avais peur de faire de la peine à Jérôme [...] maintenant, quand il y a quelque chose qui ne fonctionne pas, je lui dis doucement. C'est moi la mère et il le respecte. » À cet égard, elle précise avoir apprécié les conseils et le soutien de son éducatrice. L'approche de cette intervenante lui a permis de développer une meilleure confiance en son potentiel et a favorisé l'acquisition d'habiletés parentales. Aussi, tous les participants ressentent de la fierté face aux réussites de leurs enfants (p. ex. obtention du permis de conduire ou accès à un emploi). Ils estiment avoir, en quelque sorte, contribué à de tels succès, ce qui amplifie leur sentiment de fierté.

Une motivation importante à exercer ses droits et à répondre à ses obligations vis-à-vis de leur enfant de manière autonome et responsable émerge des propos des parents rencontrés. Par exemple, depuis qu'elle est mère, Fannie a l'impression que ses choix et son autonomie sont davantage respectés par ses proches et par les professionnels impliqués dans sa vie. Le témoignage d'Alice va dans le même sens :

On se sent valorisés beaucoup, beaucoup. On trouve que le monde aussi s'intéresse à nous par rapport à Jérôme et on voit d'autres parents qui passent près de nous et on a la tête haute en me disant : « Moi, je fais ça avec mon gars! » La prof, elle m'a dit qu'il y avait plein de parents qui ne s'occupaient pas de leur enfant. Mais moi, je prends de l'intérêt à Jérôme.

Leur désir d'autonomie ne s'oppose pas à l'éventualité d'accepter de l'aide extérieure. En effet, ils ont une ouverture à recevoir des services leur permettant d'améliorer leurs habiletés parentales et de mieux soutenir la réussite scolaire et sociale de l'enfant. Dans le cas de Marie, cette ouverture à l'aide extérieure se traduit par une forme de désinvestissement au regard de certaines fonctions liées à l'exercice parental (p. ex. gestion des comportements), lesquelles sont toujours assumées par ses propres parents. Elle dit apprécier ce transfert d'autorité et ne pas avoir l'impression d'être substituée.

Même s'ils vivent grâce aux prestations gouvernementales, ces parents n'hésitent pas à engager des dépenses visant, de leur point de vue, l'amélioration de la qualité de vie de l'enfant. Alice rapporte débourser une partie considérable de son budget afin que son fils puisse fréquenter une école de meilleure réputation. Pour sa part, Fannie, dont la fille est âgée de 4 ans, précise : "Toutes les fois qu'il y a des activités à la garderie, elle y va. Je lui paye toutes ses activités. » Tous les parents ont évoqué prendre plaisir à réaliser des activités de loisirs avec leur enfant (p. ex. cinéma, quilles, restaurant, etc.), et ce, même si elles entraînent des dépenses importantes. 
Pour certains, un tel investissement découle d'une volonté de s'assurer que leur enfant ne manque de rien et qu'il ne soit pas exposé aux mêmes difficultés qu'ils ont vécues étant jeunes. Pour une des mères, cet investissement est également motivé par le désir de garder ses enfants auprès d'elle. À cet égard, elle rapporte l'expérience douloureuse du signalement de ses enfants pour des motifs de violence et de négligence. Elle mentionne qu'elle avait alors fait preuve d'ouverture à l'idée de collaborer avec les intervenants en protection de la jeunesse et de détermination à adopter les mesures proposées. Au moment de l'entretien, ses enfants demeuraient toujours avec elle.

\subsubsection{Attitude générale et facteurs d'influence}

Une attitude générale plutôt favorable à l'égard de la parentalité des personnes présentant une DI/LI se dégage des propos des cinq participants. Ils estiment que la plupart d'entre elles sont généralement capables d'exercer leur rôle parental lorsqu'elles reçoivent le soutien qui répond à leurs besoins. Cependant, considérant l'investissement considérable et continu, Léa et Fannie sont d'avis que ce ne sont pas tous les adultes présentant une DI/LI qui ont les capacités nécessaires pour exercer ce rôle. Elles leur recommandent de réfléchir longuement avant de concrétiser leur désir d'enfant.

Certains facteurs semblent exercer une influence favorable sur l'attitude générale des cinq répondants. Sur ce plan, ils ont tous évoqué les retombées positives d'une relation établie avec un intervenant de confiance depuis plusieurs années (plus de 10 ans). Aussi, trois parents peuvent compter sur un réseau de soutien informel composé de membres de la famille élargie et de nombreux amis. D'autres ont un réseau plus restreint; c'est le cas de celui d'Alice et de Marc, lequel se limite au conjoint. Néanmoins, ce couple marié s'estime comblé par leur relation fondée sur l'amour, le respect, le soutien à travers des étapes de vie difficiles et le partage des tâches misant sur les forces de chacun. À ce sujet, Marc précise : «Avec Alice, ça va très bien. [Après l'école], elle lui montre ses études, vu que moi je ne sais quasiment pas lire et écrire. Pendant ce temps-là, je cuisine et je fais la vaisselle [...] On se complète comme il le faut. » Ces deux parents ont des frères et des sœurs qu'ils fréquentent très rarement, ce qu'ils justifient par l'éloignement géographique.

Tous les répondants justifient l'actualisation de leur rêve de devenir parent par leur amour pour les enfants. Cet amour semble alimenté par les modèles parentaux avec qui ils ont été en contact et sur lesquels ils prennent appui dans la construction de leur parentalité.

\subsubsection{Connaissances}

Tous les répondants ont évoqué la présence de modèles parentaux sur lesquels ils prennent appui dans l'exercice de leur rôle parental. Pour certains, l'inspiration vient des façons de faire de leurs propres parents, qu'ils reconnaissent comme ayant été bons pour eux. Pour d'autres, au contraire, leur passé qualifié de douloureux est mis de côté pour s'appuyer plutôt sur les modèles actuels offerts par des amis et des professionnels. Dans le cas de Fannie, par exemple, une relation de proximité s'est tissée entre elle et une intervenante qu'elle voit interagir régulièrement avec ses propres filles.

Trois rôles principaux ressortent des propos des répondants. Leur conception de ces rôles permet d'entrevoir les connaissances sur lesquelles ils s'appuient. Ces rôles renvoient à la réponse aux besoins primaires, à l'éducation et à la protection. Pour assumer adéquatement les responsabilités supposées, surtout après la naissance de l'enfant et lors des périodes de transition, telles que l'entrée à l'école, les parents rencontrés estiment avoir besoin d'être soutenus dans l'acquisition de nouvelles 
habiletés. Comme le mentionne Léa : «Tout est apprendre du rôle de la mère. Même si avant je gardais, là, ce n'est pas pareil, parce que c'est le tien. »

Pour les répondants, être parent comporte une composante éducative importante qui se traduit, notamment, par la nécessité de donner de petites responsabilités à son enfant pour favoriser son autonomie et par l'accompagnement aux devoirs, une tâche essentielle pour deux des mères interviewées. Alice s'y adonne avec constance, rigueur, assiduité et assurance, alors que Fannie, qui a fréquenté des classes spéciales, appréhende le fait qu'elle ne puisse accompagner son enfant très longtemps dans son cheminement scolaire.

Une grande partie du discours des cinq répondants porte sur la protection de l'enfant. Ceci s'actualise, entre autres, par la prévention du danger grâce à une surveillance constante de l'enfant. À cet égard, Alice indique son inquiétude vis-à-vis des sorties de son fils de 8 ans à l'extérieur de la maison : « Je ne suis pas capable de le laisser aller. Je ne veux pas le laisser aller tout seul pour tout de suite, parce que j'ai peur. Lui, il parle à tout le monde, et j'ai peur de ça. On le surveille et on le suit de près. » Par ailleurs, les parents qui ont subi des mauvais traitements et de la violence pendant leur enfance semblent avoir une crainte plus prononcée du danger. Par exemple, Fannie interdit à son enfant de s'éloigner d'elle lors des visites au parc. Alice exprime ressentir de l'inquiétude à l'idée que son enfant ait de la difficulté à s'intégrer à l'école et à se faire des amis. En vue de l'aider, elle communique souvent avec l'enseignante et demande des conseils pour favoriser son acceptation par les pairs.

\subsection{Représentations de la parentalité des parents ayant perdu la garde}

Les résultats de cette section renvoient aux quatre parents ayant perdu la garde de leur enfant. Cette situation s'est produite soit à la naissance, soit lorsque les enfants étaient âgés de moins de deux ans. Même si leur enfant leur a été retiré par les autorités légales, Caroline et Vicky précisent qu'elles ont un droit de visite et qu'elles peuvent voir leur enfant lors de contacts supervisés par un intervenant de la protection de la jeunesse. Pour sa part, Martin rencontre sa fille à intervalles fixes, sous la supervision de sa belle-mère. Philippe jouit d'un accord amical avec les grands-parents maternels chez qui demeure son fils depuis l'âge de trois ans. Cette entente a permis la fermeture du dossier dans le système de protection de la jeunesse. Depuis, père et fils passent toutes les fins de semaine ensemble et partagent des moments de plus longues durées lors des vacances d'été.

\subsubsection{Image évocatrice}

Pour ces quatre parents, l'objet d'étude évoque des sentiments opposés, distinctifs de leur représentation bien singulière. D'une part, la parentalité symbolise fierté et d'autre part, elle suscite tristesse, frustration, amertume et rejet. Cette situation n'est pas étrangère au fait que leurs responsabilités parentales sont déléguées à d'autres adultes partageant le quotidien des enfants. En fait, pour la plupart, la parentalité passe essentiellement par la relation qu'ils entretiennent avec les intervenants de la protection de la jeunesse et, à cet égard, leurs expériences sont plutôt négatives.

D'abord, dès le départ, ils ont eu de la difficulté à comprendre le processus imposé; ils estiment que leur rythme et leur compréhension des dispositions de la Loi de la protection de la jeunesse (LPJ) n'ont pas été respectés. Relatant une expérience au tribunal, Vicky rapporte que tout va trop vite et que «c'est difficile à comprendre comment les phrases sont formulées ». Martin et son épouse, quant à eux, ont réalisé, quelques jours seulement après leur rencontre avec l'intervenant, qu'ils venaient de consentir à l'adoption de leur enfant. Le processus a pu être révoqué grâce aux démarches légales intentées par lui, son intervenante de confiance (organisme 
communautaire) et un avocat. Cette situation traduit bien la vulnérabilité de ces parents lorsque la portée de leurs décisions ne leur est pas expliquée avec soin.

D'ailleurs, tous estiment qu'il y a eu un complot organisé contre eux pour que la garde de leur enfant leur soit enlevée. Caroline exprime qu'elle s'est sentie « embobinée » et "sous la menace» de signer des papiers qu'elle ne comprenait pas. Ses propos témoignent aussi d'une incompréhension des motifs du retrait de leur enfant, ce qui caractérise également la situation de Vicky. Ces dernières éprouvent toujours un sentiment d'injustice et de l'amertume. Philippe raconte qu'il a dû passer une évaluation psychologique, à la demande du tribunal, laquelle était, de son avis, biaisée dès le départ: "La psychologue qu'ils ont prise, dit-il, c'était une ancienne cliente de mon beau-père. Alors, elle était achetée. Pour eux, j'étais un gars violent qui criait tout le temps. Alors, le juge, il y est allé.» De plus, ces parents sont convaincus de la malhonnêteté de certains professionnels en protection de la jeunesse qui leur auraient caché des informations et qui auraient menti lors de la rédaction du rapport ayant mené au retrait de la garde. Même actuellement, Vicky rapporte se sentir observée et jugée lorsqu'elle visite son fils dans un local supervisé par une travailleuse sociale (TS). Bien que sa plus jeune fille soit placée depuis plusieurs années, Caroline a toujours l'impression d'être constamment surveillée, critiquée et jugée par la TS qui encadre ses contacts périodiques avec son enfant. Elle l'exprime ainsi : «Je lui achète du linge, je lui donne de l'argent, je l'amène manger au restaurant. Ça me fait plaisir, mais là, l'intervenante, elle me dit: "Tu n'es pas obligée, là, elle a tout ce qui lui faut dans la famille d'accueil." Et je lui dis : "Eh! je suis sa mère, je fais ce que je veux." »

L'impression d'avoir été évalué comme n'ayant pas les compétences parentales requises en raison de leur DI est explicitement nommée par Martin. Lors d'une rencontre visant à l'informer des décisions du tribunal, l'intervenante se serait adressée exclusivement à son accompagnatrice et aurait utilisé des termes techniques juridiques relevant pour lui d'un « jargon » incompréhensible. Le sentiment d'injustice et la frustration qu'il a éprouvés à la suite de cet évènement, jumelés à la souffrance d'être séparé de son enfant, l'ont amené à exprimer ouvertement sa colère lors des contacts avec les intervenants de la protection de la jeunesse : « Les premières fois, il y avait trois agents de sécurité sur le bord de la porte à cause que j'étais très violent et très vulgaire, selon eux [...] Mais moi [j'étais choqué] par rapport au fait qu'ils m'ont ôté mes visites, ils m'ont enlevé mes heures de téléphone. » Ce sentiment d'être incompris et stigmatisé est aussi partagé par Philippe, qui raconte : «Eux autres, ils ont convenu que j'étais un gars violent, que je criais tout le temps. Ce qu'ils ne savent pas aussi, c'est que ma mère est presque sourde. Il faut parler fort, elle est sourde à $96 \%$. [...] Alors, ce n'est pas parce que je suis enragé, c'est parce que j'ai pris une habitude. »

Aussi, un point que ces quatre parents partagent en commun est l'absence d'un réseau de proximité soutenant et impliqué dans le partage des responsabilités parentales au moment du retrait de la garde. Martin se sent méprisé par sa belle-mère qui lui renvoie une image fort négative du père qu'il est. Il rapporte ses paroles lorsqu'elle a appris la grossesse de sa fille à l'époque: "Elle m'a dit: "Tu vas mettre ton enfant dans la misère!" » Après la naissance de l'enfant, le couple s'est senti envahi par ses fréquentes visites caractérisées par des chicanes. Pour leur part, Philippe et sa conjointe ont été tenus à distance par la grand-mère paternelle. Celle-ci aurait accaparé le bébé jusqu'à l'âge de trois ans, évoquant le fait que ses parents ne seraient jamais capables de s'en occuper.

Paradoxalement, la fierté est aussi évoquée à maintes reprises par les parents lorsqu'ils sont amenés à discuter de leur relation avec leur enfant. Ils énumèrent les qualités de leur enfant qui les rendent fiers (p. ex. sociable, intelligent, beau) et racontent des 
souvenirs les mettant en valeur. Ils parlent aussi de leurs progrès en matière de développement (p. ex. motricité améliorée), d'apprentissages (p. ex. savoir compter) et de résultats scolaires. La tranquillité d'esprit est également mentionnée. À cet égard, Caroline explique : « Ça ne me dérange pas finalement qu'elle soit placée, parce qu'elle est bien. Elle est bien, très bien chez Madame. » Pour Philippe, la présence de son enfant le motive à développer une meilleure gestion de sa colère et à communiquer ses mécontentements de manière plus appropriée. Toutefois, évoquer de tels aspects positifs fait ressurgir un sentiment de tristesse d'être séparés de leur enfant. Cette tristesse est ressentie avec plus d'intensité à deux moments particuliers, soit lors de la séparation après un heureux moment passé avec leur enfant et quand ils se rendent compte qu'ils ne peuvent pas aider leur enfant lorsque celui-ci vit des difficultés. De leur point de vue, leur enfant désire vivre auprès d'eux. Caroline explique : "Elle est très bien là-bas [famille d'accueil], mais elle, elle n'aime pas ça, elle aimerait ça revenir avec moi. »

\subsubsection{Attitude générale et facteurs d'influence}

L'attitude générale des quatre participants à l'égard de l'objet de représentation est caractérisée par l'ambivalence. Trois participants sont d'avis que l'exercice de la parentalité par des adultes présentant une DI/LI est possible, mais qu'il nécessite la mise en place de mesures de soutien (réseau formel ou informel) considérables. De leur point de vue, les mesures proposées par le système public de services sont soit inexistantes, insuffisantes ou mal adaptées aux besoins de ces personnes. Philippe et Martin leur conseillent de réfléchir longuement et de se demander si toutes les conditions favorables sont réunies. Philippe regrette de ne pas avoir fait cette réflexion. Il conclut : «Si c'était à refaire, je n'en aurais pas de Kevin. Surtout avec ce que j'ai vécu, si c'était à refaire, je n'en aurais pas. »

À travers les évènements difficiles, quelques personnes significatives de la famille proche ou élargie (p. ex. frère, belle-sœur) ont néanmoins assuré une présence soutenante aux quatre parents interrogés. L'appui offert leur a permis de développer une attitude plus positive à l'égard de la parentalité. À ce sujet, Philippe et Martin comptent sur un réseau d'amis solide. Ils se retrouvent souvent grâce aux activités offertes par un organisme communautaire dont les membres présentent tous une DI/LI. Philippe s'explique : «On est comme une fraternité : on va tous s'aider les uns les autres. S'il y a un membre dans le besoin, on va tous être là pour l'aider. » De surcroît, Philippe, Martin et Vicky jouissent d'une relation de proximité avec une intervenante d'un organisme communautaire de leur région. Ils y ont puisé écoute, soutien et considération positive. Ils ont également été accompagnés lors des différentes rencontres convoquées par les professionnels de la protection de la jeunesse. Des actions relevant de la défense des droits ont été réalisées par cette intervenante dans la situation des deux pères, ce qu'ils ont apprécié. Celle-ci les a également aidés à prendre du recul et à tempérer leurs réactions afin de pouvoir conserver un lien avec leur enfant.

\subsubsection{Connaissances}

Peu de propos font référence aux connaissances de ces répondants à l'égard de l'objet d'étude. Ils conçoivent que l'exercice des droits et des devoirs parentaux implique un investissement personnel et financier important pour tout parent. À ce sujet, Philippe justifie le placement de son enfant par l'insuffisance de ses ressources matérielles. La situation de Martin est différente : la nécessité de gagner de l'argent exigeait qu'il s'absente de la maison et sa conjointe était incapable d'assumer seule les soins à leur enfant. 
Martin et Philippe estiment que la volonté de fonder une famille unie et « d'assurer la relève » a alimenté leur désir d'enfant. De leur point de vue, son actualisation aurait été impulsive; ils ont ignoré les tenants et les aboutissants des responsabilités découlant de la concrétisation de ce désir. Martin ajoute que son désir est devenu plus fort lorsque son frère aîné a eu son fils. Pour sa part, Vicky qualifie sa grossesse « d'accident de parcours ». Néanmoins, elle exprime le désir d'avoir un autre enfant dans le futur. Caroline a souhaité devenir mère pour combler un vide affectif, comme l'illustrent ses propos : «Pourquoi j'ai décidé de devenir maman? Parce que je voulais arrêter de me sentir seule et je voulais avoir quelqu'un dans ma vie que j'aime et que je réconforte. » Vicky a des propos similaires à ce sujet: "D'être maman, ça me fait sentir bien parce que tu ressens de l'amour par quelqu'un pour qui tu as de l'importance. Selon moi, quand tu as un enfant, tu sais que tu as une présence. Il peut toujours être là quand tu es triste ou quoi que ce soit. »

\section{Discussion}

D’après les analyses des propos recueillis dans cette étude, la parentalité symbolise l'accès à un rôle hautement désiré et à une image de soi positive pour les parents rencontrés. Une telle représentation a été relevée par d'autres chercheurs (Edmonds, 2000; Mayes et al., 2011). En effet, être parent confère un statut d'adulte responsable, digne d'assumer pleinement son autonomie. Son accès peut symboliser l'occasion de prouver ses compétences à autrui. C'est comme si l'identité parentale venait gommer "l'identité stigmatisée» (Booth et Booth, 1995; Mercier, 2004) des personnes présentant une DI, une identité souvent dévalorisée et jugée inférieure par autrui (Croizet et Leyens, 2003). Selon Beart et al. (2005), l'étiquette de la DI a une portée puissante et dominante sur l'identité primaire de la personne qui la porte. En effet, son poids peut parfois anéantir l'importance accordée aux identités liées au genre (Burns, 2000), à l'orientation sexuelle, à la religion ou à l'origine ethnique (Walmsley et Downer, 1997).

Sans nier leurs difficultés sur le plan de l'apprentissage ou de la compréhension, il est intéressant de constater que les répondants se comparent tous à des parents sans DI/LI. Ils s'estiment tout aussi bons, et parfois même meilleurs qu'eux. Selon Croizet et Leyens (2003), les personnes stigmatisées ont davantage tendance à utiliser des stratégies visant la protection de leur estime de soi lorsque leur statut social est menacé. À titre d'exemple, elles peuvent s'identifier à un groupe social plus avantagé et faire le choix de cibles de comparaison jugées inférieures pour rehausser leur image de soi.

Devenir parent peut susciter l'émergence de souvenirs douloureux pour les adultes ayant eu une enfance difficile. Dans cette étude, ce passé semble avoir motivé les répondants à offrir à leur enfant ce qui leur a manqué en s'appuyant sur de bons modèles. Selon Leroux et Scelles (2007), ces parents ont tout à réinventer de «l'être parent », attentif aux peines, aux difficultés, aux besoins et aux désirs de son enfant. Ils doivent aussi tenter d'interpréter sa pensée afin que celui-ci se sente en sécurité et en confiance. Ceci représente un défi de taille lorsque sont considérées les limites du fonctionnement adaptatif associées à la DI/LI. Selon les propos de la plupart des participants à cette recherche, la présence de l'enfant apparaît une source puissante de motivation à développer les compétences parentales nécessaires à son bien-être.

Les résultats mettent également en lumière les répercussions négatives de la perte de la garde sur l'identité parentale des répondants concernés, un constat qui rejoint ceux qui ont été établis dans le cadre de recherches menées auprès de populations de parents sans DI/LI (Euillet et Zaouche-Gaudron, 2008; Sécher, 2010). Après la 
séparation, les répondants ont eu à donner un sens nouveau à leur parentalité, dans un moment caractérisé par une perte d'estime de soi. Dans le regard de membres de leur entourage et de professionnels de la protection de la jeunesse, ils ont perçu une disqualification et un manque de reconnaissance de leur potentiel à élever leurs enfants. Leur représentation du rôle parental qu'ils peuvent exercer dans un tel contexte et les relations avec les acteurs impliqués ont une incidence majeure sur leur parentalité (Euillet et Zaouche-Gaudron, 2008). Pour Barreyre, Peintre et Fiacre (2002), la qualité du lien entre les parents et les intervenants en protection a une influence déterminante sur l'implication du parent dans la vie de l'enfant. Le sentiment de disqualification accentuerait le risque que les parents séparés de leur enfant démissionnent ou qu'ils "partent en guerre » contre ces professionnels (Savourey, 2002). Il s'agit là de «moyens projectifs de défense qui limitent la culpabilité [...] à la mesure de leur fragilité et de leur souffrance » (Euillet et Zaouche-Gaudron, 2008: 19). Dans cette étude, lorsque les participants concernés explorent les motifs qui ont conduit au placement de leur enfant, ceux-ci renvoient à des causes externes, qui échappent à leur contrôle. Selon Vallerand (2006), une telle attribution a pour but de protéger l'estime de soi et la santé mentale des individus. À ce sujet, Edmonds (2000) met en exergue les conséquences néfastes pouvant survenir lorsque l'identité parentale des parents présentant une DI est attaquée. Ces conséquences peuvent se traduire par du repli sur soi, de l'isolement, une baisse de l'estime de soi et de la détresse psychologique.

Dans la quête de l'appropriation ou du maintien d'une identité parentale positive et d'une représentation favorable de leurs compétences parentales, l'accompagnement d'un intervenant significatif apparaît essentiel pour la quasi-totalité des parents. L'engagement dans un organisme communautaire, le soutien d'amis et de membres de la famille élargie et la présence d'une conjointe ou d'un conjoint soutenant représentent autant de facteurs qui ont des répercussions positives sur le parcours de vie de ces parents.

\section{Limites et considérations méthodologiques}

Les résultats de cette étude exploratoire doivent être interprétés à la lumière de ses limites liées aux caractéristiques des participants, aux choix méthodologiques et à la réalisation des entretiens. Relativement à l'échantillon, les parents séparés de leurs enfants ont tous perdu la garde lorsque ceux-ci étaient en bas âge. Pour bonifier les analyses, il aurait été intéressant de recruter des informateurs ayant vécu cette situation lorsque l'enfant était d'âge scolaire ou devenu adolescent. Aussi, plus de la moitié des répondants $(n=5)$, recrutés au sein d'un même organisme communautaire, se connaissaient entre eux. Parmi les autres participants se retrouve aussi un couple. Il est possible que les échanges entre ces parents aient influencé leurs propos lors des entretiens. Ceci aurait pu être évité par l'ajout d'un critère d'exclusion de l'échantillon à cet effet en amont du recrutement.

Par ailleurs, lorsque les participants présentent des limitations intellectuelles, la motivation à répondre serait plus souvent marquée par la désirabilité sociale, et ce, surtout lors des entretiens face à face (Carrier et Fortin, 1994). Selon Guillemette et Boisvert (2003), parfois par peur d'être jugés, certains d'entre eux auraient davantage tendance à fournir des réponses qui semblent correspondre aux attentes perçues chez leur interlocuteur pour en retirer de l'approbation et de la valorisation. De plus, l'hétérogénéité de leurs habiletés cognitives et langagières favorise le risque de surévaluation du niveau de compréhension des questions par l'intervieweur lorsque le participant semble détenir de bonnes habiletés d'expression (Finlay et Lyons, 2002). 
La moindre efficience de leur fonctionnement intellectuel peut aussi complexifier la tâche de répondre à des questions faisant appel à leur mémoire. Lors de la collecte de données de cette recherche, plusieurs précautions ont été prises en considération des caractéristiques des participants sur le plan de la construction du discours, de la cognition et des relations interpersonnelles. Elles se sont actualisées par : l'emploi de questions ouvertes pour contourner la plus forte tendance à l'acquiescement (Carrier et Fortin, 1994); la recherche d'un discours plus narratif et descriptif ponctué par des demandes d'ajouts et non d'explications; la reformulation des questions; l'emploi du vocabulaire du participant; la référence à des termes concrets; l'attention soutenue aux réactions semblant manifester la compréhension; une durée plus courte des entretiens ainsi que la valorisation verbale et non verbale des réponses des interviewés. Cinq informateurs ont souhaité être accompagnés de leur témoin de confiance lors des entretiens. Leur choix était motivé par le souhait d'être soutenus, craintifs de vivre une perte de mémoire ou un moment de tristesse dus à l'évocation d'un souvenir malheureux. Il demeure impossible de déterminer si cette présence a limité ou favorisé une communication optimale de leurs propos.

Finalement, il est possible que la prégnance de composantes affectives négatives (tristesse et souffrance) ait orienté le discours de certains parents vers une centration sur les aspects plus douloureux, laissant sous silence d'autres éléments propres à la complexité de leur expérience. Le passage du temps et la présence de difficultés limitant l'expression de leurs sentiments ont également pu teinter leurs souvenirs ou leur en faire oublier des composantes importantes.

\section{Conclusion}

Les résultats de cette étude ont permis l'exploration des représentations sociales de la parentalité des personnes vivant avec une DI/LI chez des parents présentant ce profil. La démarche de recherche qualitative déployée a permis d'accéder aux points de vue des principales personnes concernées, par l'accès à leur expertise, à leurs contextes respectifs ainsi qu'à la signification qu'ils attribuent à leurs actions (Comeau, 1994; Kvale, 2009; Savoie-Zajc, 2009). L'hétérogénéité relative des caractéristiques des répondants et de leurs expériences personnelles a enrichi la lecture du phénomène à l'étude par la considération de différents aspects qui leur sont propres (Rubin et Babbie, 2013; Yegidis et Weinbach, 2006).

Dans la perspective d'approfondir le sujet de cette étude, il serait intéressant de réaliser des analyses plus poussées, guidées par un devis de recherche mixte, en sollicitant un échantillon plus important de parents. Aussi, l'exploration des facteurs ayant contribué à l'adaptation résiliente de certains parents présentant une DI/LI pourrait s'avérer une avenue porteuse de sens dans la création de conditions favorables à l'exercice d'une parentalité positive et épanouie.

Les résultats de l'étude présentés dans cet article mettent en lumière l'importance fondamentale que ces parents attribuent au soutien dans le succès de leur projet parental et dans le développement d'une identité parentale positive. Les principaux obstacles relevés semblent provenir, en majeure partie, des représentations défavorables perçues chez leurs proches et chez les intervenants de la protection de la jeunesse. Il semble impératif que des efforts de sensibilisation, d'éducation et de conscientisation soient investis afin que ces parents jouissent des mêmes possibilités de participation sociale que n'importe quel autre citoyen ou citoyenne, et ce, même si leurs particularités individuelles génèrent d'importants besoins en termes de soutien et d'accompagnement (Fougeyrollas, 2009). 


\section{Remerciements}

Cet article fait partie de la thèse de doctorat en service social de la première auteure. Nous tenons à remercier les parents ayant participé à ce projet ainsi que les organisations suivantes pour leur soutien financier et leur collaboration : le Fonds de recherche du Québec-société et culture (FQRSC); le Consortium National de Recherche sur l'Intégration Sociale (CNRIS); le Centre jeunesse de Québec-Institut universitaire (CJQ-IU) ainsi que le Comité d'éthique de la recherche conjoint destiné aux centres de réadaptation en déficience intellectuelle et en troubles envahissants du développement (CÉRC/CRDITED).

\section{Références}

Abric, J.-C. 1994. Pratiques sociales et représentations, Paris, Presses universitaires de France.

Abric, J.-C. 2003. Méthodes d'étude des représentations sociales, Ramonville Saint-Agne, Érès.

Abric, J.-C. 2009. "L'étude expérimentale des représentations sociales », dans Les représentations sociales, $7 \mathrm{e}$ édition, sous la dir. de D. Jodelet, Paris, Presses universitaires de France, p. 205-223

Aunos, M. et M. Feldman. 2002. "Attitudes towards sexuality, sterilization and parenting rights of persons with intellectual disabilities », Journal of Applied Research in Intellectual Disabilities, vol. 15, p. 285-296.

Aunos, M. et M. Feldman. 2010. "Assessing parenting capacity in parents with intellectual disabilities», dans Enfants à protéger, parents à aider: des univers à rapprocher, sous la dir. de C. Chamberland, S. Léveillé et N. Trocmé, Québec, Presses de l'Université du Québec, p. 223 à 240.

Bardin, L. 2003. "L'analyse de contenu et de la forme des communications », dans Les méthodes des sciences humaines, sous la dir. de S. Moscovici et F. Buschini, Paris, Presses universitaires de France, p. 243-269.

Bardin, L. 2007. L'analyse de contenu, Paris, Presses Universitaires de France.

Barreyre, J.-Y., C. Peintre et P. Fiacre. 2002. La parole aux usagers 2. Enquête auprès des parents d'enfants placés des Yvelines : conseil général des Yvelines, Rhône-Alpes, Carrefour des acteurs de l'action sociale et médico-sociale.

Beart, S., G. Hardy et L. Buchan. 2005. « How people with intellectual disabilities view their social identity: a review of the literature ", Journal of Applied Research in Intellectual Disabilities, vol. 18, no 1, p. 47-56.

Blais, M. et S. Martineau. 2006. "L'analyse inductive générale : description d'une démarche visant à donner un sens à des données brutes", Recherches Qualitatives, vol. 26, no 2, p. 1-18.

Booth, T. et W. Booth. 1995. "Unto us a child is born: the trials and rewards of parenthood for people with learning difficulties », Australia and New Zealand Journal of Developmental Disabilities, vol. 20, no 1, p. 25-39.

Booth, T. et W. Booth. 1998. « Think of the children: growing up with parents who have learning difficulties », Journal of Learning Disabilities, vol. 2, no 3, p. 138-143. 
Booth, T. et W. Booth. 2004. «Brief research report: findings from a court study of care proceedings involving parents with intellectual disabilities", Journal of Policy in Intellectual Disabilities, vol. 1, no 3/4, p. 179-181.

Booth, T. et W. Booth. 2005. " Parents with learning difficulties in the child protection system: experiences and perspectives », Journal of Intellectual Disabilities, vol. 9, no 2, p. 109-129.

Booth, T., D. McConnell et W. Booth. 2006. « Temporal discrimination and parents with learning difficulties in the child protection system », British Journal of Social Work, vol. 36, no 6, p. 997-1015.

Burns, J. 2000. «Living on the edge: women with learning disabilities », dans Women's health: contemporary international perspectives, sous la dir. de J. Ussher, London, WileyBlackwell, p. 196-203.

Carrier, S. et D. Fortin. 1994. «La valeur des informations recueillies par entrevues structurées et questionnaires auprès des personnes ayant une déficience intellectuelle : une recension scientifique», Revue Francophone de la Déficience Intellectuelle, vol. 5, no 1, p. 29-41.

Chatroussat, S. 2011. «La parentalité des personnes déficientes intellectuelles : entre stigmatisation et intégration », Dialogue, vol. 4, p. 57-67.

Comeau, Y. 1994. L'analyse des données qualitatives, Montréal, Centre de recherche sur les innovations sociales.

Coppin, B. 2001. "Déficience intellectuelle et parentalité », Revue Francophone de la Déficience Intellectuelle, vol. 12, no 2, p. 243-257.

Coppin, B. 2003. "Être parent avec une déficience intellectuelle : sortir des représentations stigmatisantes », Informations Sociales, no 112, p. 70-77.

Coppin, B. 2004. "Être parent avec une déficience intellectuelle», Pratiques Psychologiques, vol. 10, p. 25-38.

Coppin, B. 2007. «Être parent et en situation de handicap: des idées reçues à quelques réalités », Reliance, vol. 26, no 4, p. 88-96.

Croizet, J.-C. et J.-P. Leyens. 2003. Mauvaises réputations: réalités et enjeux de la stigmatisation sociale, Paris, Armand Colin.

Deconchy, J.-P. 2012. "Systèmes de croyances et représentations idéologiques », dans Psychologie sociale, 2e édition, sous la dir. de S. Moscovici, Paris, Presses Universitaires de France, p. 331-356.

Edmonds, J. 2000. " On being a mother: a positive identity in the face of adversity », Clinical Psychology Forum, vol. 137, p. 21-25.

Ehlers-Flint, M. L. 2002. "Parenting perceptions and social supports of mothers with cognitive disabilities », Sexuality and Disability, vol. 20, no 1, p. 29-51.

Euillet, S. et C. Zaouche-Gaudron. 2008. Des parents en quête de parentalité : l'exemple des parents d'enfants accueillis à l'aide sociale à l'enfance. http://sejed.revues.org/2703

Feldman, M. 1994. «Parenting education for parents with intellectual disabilities: a review of outcome studies», Research in Developmental Disabilities, vol. 15, p. 299-332.

Feldman, M. 2002. "Parents with intellectual disabilities: impediments and supports », dans Ethical dilemmas: sexuality and developmental disability, sous la dir. de D. M. 
Griffiths, D. Richards, P. Fedoroff et S. L. Watson, Kingston, An association for persons with developmental disabilities and mental health needs, p. 255-292.

Feldman, M. 2010. "Parenting education programs», dans Parents with intellectual disabilities: past, present and futures, sous la dir. de G. Llewellyn, R. Traustadóttir, D. McConnell et H. B. Sigurjónsdóttir, West Sussex, John Wiley and sons, p. 121-136.

Finlay, W. M. L. et E. Lyons. 2002. «Acquiescence in interviews with people who have mental retardation », Mental Retardation, vol. 40, no 1, p. 14-29.

Flament, C. et M.-L. Rouquette. 2003. Anatomie des idées ordinaires : comment étudier les représentations sociales, Paris, Armand Colin.

Fortin, M.-F. 2006. Fondements et étapes du processus de recherche, Montréal, Chenelière-Éducation.

Fougeyrollas, P. 2009. "Construire le sens de la participation sociale », dans Participation et responsabilités sociales, sous la dir. de V. Guerdan, G. Petitpierre, J.-P. Moulin et M.-C. Haelewyck, Berne, Peter Lang, p. 115-122.

Gray, G. 2011. «Family matters: Working with parents with learning disabilities », dans Learning disability today: the essential handbook for carers, service providers, support staff and families, 3e édition, sous la dir. de S. Carnaby, Pavilion, Brighton Publishig Ltd, p. 199-212.

Guillemette, F. et D. Boisvert. 2003. "L'entrevue de recherche qualitative avec des adultes présentant une déficience intellectuelle», Recherches Qualitatives, vol. 23, p. $15-26$.

Guinea, S. M. 2001. "Parents with a learning disability and their views on support received: A preliminary study ", Journal of Learning Disabilities, vol. 5, no 1, p. 43-56.

Herzlich, C. 1972. «La représentation sociale», dans Introduction à la psychologie sociale, sous la dir. de S. Moscovici, Paris, Larousse, p. 303-325.

Houzel, D. 1999. Les enjeux de la parentalité. Ramonville Saint-Agne, Érès.

Houzel, D. 2002. "Les enjeux de la parentalité», dans La parentalité : défi pour le troisième millénaire sous la dir. de L. Solis-Ponton, Paris, Presses universitaires de France, p. 61-70.

Jodelet, D. 2009. Les représentations sociales, 7e éd., Paris, Presses Universitaires de France.

Jodelet, D. 2012. "Représentation sociale: phénomènes, concepts et théorie », dans Psychologie sociale, 2e édition, sous la dir. de S. Moscovici, Paris, Presses universitaires de France, p. 357-378.

Julien-Gauthier, F., C. Jourdan-Ionescu et J. Héroux. 2009. «Favoriser la participation des personnes ayant une déficience intellectuelle lors d'une recherche », Revue Francophone de la Déficience Intellectuelle, vol. 20, p. 178-188.

Kvale, S. 2009. InterViews : an introduction to qualitative research interviewing, 2 éd., Thousands Oaks, SAGE publications.

Lalande, D., L. S. Éthier, C. Rivest et M. Boutet. 2002. "Parentalité et incapacités intellectuelles: Une étude pilote », Revue Francophone de la Déficience Intellectuelle, vol. 13, no 2, p. 133-154.

Lamour, M., et Barraco, M. (1998). Souffrances autour du berceau. Paris, Gaëtan Morin Éditeur Europe. 
Laperrière, A. 1997. "Les critères de scientificité des méthodes qualitatives », dans Recherche qualitative: enjeux épistémologiques et méthodologiques, sous la dir. de J. Poupart, L.-H. Groulx, J.-P. Deslauriers, A. Laperrière, R. Mayer et A. P. Pires, Montréal, Gaëtan Morin Éditeur, p. 392-418.

Ledain, M. 2008. "Parentalité, non parentalité, désir », dans Parentalité des personnes déficientes mentales, sous la dir. de M. Mercier et G. Brazier, Namur, Presses universitaires de Namur, p. 59-62.

Leroux, V. et R. Scelles. 2007. «Ce que disent les personnes déficientes de leur parentalité », Reliance, vol. 26, no 4, p. 79-87.

Llewellyn, G. 1995. "Relationship and social support: views of parents with mental retardation/intellectual disability », Mental Retardation, vol. 33, p. 349-363.

Llewellyn, G. et M. Gustavsson. 2010. «Understanding community in the lives of parents with intellectual disabilities », dans Parents with intellectual disabilities: past, present and futures, sous la dir. de G. Llewellyn, R. Traustadóttir, D. McConnell et H. B. Sigurjónsdóttir, West Sussex, John Wiley and sons Ltd., p. 79-94.

Llewellyn, G. et D. McConnell. 2002. "Mothers with learning difficulties and their support networks », Journal of Intellectual Disability Research, vol. 46, p. 17-34.

Llewellyn, G. et D. McConnell. 2010a. "You have to proove yourself all the time: people with learning disabilities as parents ", dans Learning disability: a life cycle approach to valuing people, sous la dir. de G. Grant, P. Goward, M. Richardson et P. Ramcharan, Maidenhead, Open University Press, p. 441-467.

Llewellyn, G. et D. McConnell. 2010b. "Looking back on their own upbringing », dans Parents with intellectual disabilities: past, present and futures, sous la dir. de G. Llewellyn, R. Traustadóttir, D. McConnell et H. B. Sigurjónsdóttir, West Sussex, John Wiley and sons Ltd., p. 33-49.

Llewellyn, G., R. Traustadóttir, D. McConnell et H. B. Sigurjónsdóttir. 2010. Parents with intellectual disabilities: past, present and futures, West Sussex, John Wiley and sons Ltd.

Mariotti, F. 2003. « Tous les objets sociaux sont-ils objets de représentations sociales? Questions autour de la pertinence », Journal International sur les Représentations Sociales, vol. 1, no 1, p. 2-13.

Mayer, R. et J.-P. Deslauriers. 2000. «Quelques éléments d'analyse qualitative : l'analyse de contenu, l'analyse ancrée, l'induction analytique et le récit de vie », dans Méthodes de recherche en intervention sociale, sous la dir. de R. Mayer, F. Ouellet, M.-C. Saint-Jacques, D. Turcotte et collaborateurs, Boucherville, Gaëtan Morin, p. 159-189.

Mayes, R., G. Llewellyn et D. McConnell. 2008. «Active negotiation: mothers with intellectual disabilities creating their social support networks», Journal of Applied Research in Intellectual Disabilities, vol. 21, no 4, p. 341-350.

Mayes, R., G. Llewellyn et D. McConnell. 2011. " "That's who I choose to be": the mother identity for women with intellectual disabilities", Women's Studies International Forum, vol. 34, p. 112-120.

McConnell, D. 2008. «Parents labelled with Intellectual Disability: position of the International Association for the Scientific Study of Intellectual Disabilities Special Interest Research Group (IASSID-SIRG) on Parents and Parenting with Intellectual Disabilities », Journal of Applied Research in Intellectual Disabilities, vol. 21, p. 296-307. 
McConnell, D. et H. B. Sigurjónsdóttir. 2010. "Caught in the child protection net », dans Parents with intellectual disabilities: past, present and futures, sous la dir. de G. Llewellyn, R. Traustadóttir, D. McConnell et H. B. Sigurjónsdóttir, West Sussex, John Wiley and sons Ltd., p. 171-187.

McGraw, S. et S. Candy. 2010. «Supported decision making for women with intellectual disabilities ", dans Parents with intellectual disabilities: past, present and futures, sous la dir. de G. Llewellyn, R. Traustadóttir, D. McConnell et H. B. Sigurjónsdóttir, West Sussex, John Wiley and sons Ltd., p. 137-154.

Mercier, M. 2004. L'identité handicapée, Namur, Presses universitaires de Namur.

Ministère de la Santé et des Services sociaux. 2006. Mécanisme encadrant l'examen éthique et le suivi continu des projets multicentriques.

http://ethique.msss.gouv.qc.ca/site/fr_mecanismemulticentrique.phtml

Mirfin-Veitch, B. 2010. "Citizenship and community participation », dans Parents with intellectual disabilities: past present and futures, sous la dir. de G. Llewellyn, R. Traustadóttir, D. McConnell et H. B. Sigurjónsdóttir, West Sussex, John Wiley and sons Ltd., p. 95-106.

Moliner, P. 1993. "Cinq questions à propos des représentations sociales », Les Cahiers Internationaux de Psychologie Sociale, vol. 20, p. 5-14.

Moliner, P. 1996. Images et représentations sociales : de la théorie des représentations sociales à l'étude des images sociales, Grenoble, Presses de l'Université de Grenoble.

Moliner, P., P. Rateau et V. Cohen-Scali. 2002. Les représentations sociales : pratique des études de terrain, Rennes, Presses Universitaires de Rennes.

Moscovici, S. 1961. La psychanalyse, son image et son public: étude sur la représentation sociale de la psychanalyse, Paris, Presses Universitaires de France.

Negura, L. 2004. L'analyse de contenu dans l'étude des représentations sociales. http://sociologies.revues.org/pdf/993

Nils, F. et B. Rimé. 2003. «L'interview», dans Les méthodes des sciences humaines, sous la dir. de S. Moscovici et F. Buschini, Paris, Presses universitaires de France, p. 165-185.

Painz, F. 1993. «Parents with a learning disability », Social Work Monographs, no 116, p. 1-40.

Pasleau, J.-P. et J.-L. Collignon. 1997. "Handicap mental et représentations sociales », dans Éthique et handicap mental, sous la dir. de J. Duchêne, M. Mercier, J. Delville, M.-L. Delfosse, M. Mattys et O. Witdouck, Namur, Presses Universitaires de Namur, p. 33-39.

Pires, A. P. 1997. "Échantillonnage et recherche qualitative : essai théorique et méthodologique", dans La recherche qualitative: enjeux épistémologiques et méthodologiques, sous la dir. de J. Poupart, J.-P. Deslauriers, L.-H. Groulx, A. Laperrière, R. Mayer et A. P. Pires, Montréal, Gaëtan Morin Éditeur, p. 113-169.

Pixa-Kettner, U. 1999. "Follow-up study on parenting with intellectual disability in Germany », Journal of Intellectual \& Developmental Disability, vol. 24, no 1, p. 75-93.

Pixa-Kettner, U. 2008. « Parenting with intellectual disability in Germany: results of a new nationwide study ", Journal of Applied Research in Intellectual Disabilities, vol. 21, p. 315-319.

Poussin, G. 2004. La fonction parentale, 3e éd., Paris, Dunod.

Preston, P. 2012. Parents with disabilities.

É. Milot, D. Turcotte et S. Tétreault, Représentations sociales et parentalité : les points de vue... Enfances Familles Générations, no 22, 2015, p. 182-205 - www.efg.inrs.ca 
Rao, T. 2013. «Implementation of an intensive, home-based program for parents with intellectual disabilities », Journal of Public Child Welfare, vol. 7, no 5, p. 691-706.

Rateau, P. 2007. "Les représentations sociales », dans Psychologie sociale, 2e éd., sous la dir. de J.-P. Pétard, Paris, Éditions Bréal, p. 164-219.

Robin, M. et A.-M. Fontaine. 2013. «Transitions familiales et construction des identités parentales », La Revue Internationale de l'Éducation Familiale, no 33, p. 11-15.

Rubin, A. et E. R. Babbie. 2013. Essential research methods for social work, 3e éd., Belmont, Brooks/Cole, Cengage Learning.

Savoie-Zajc, L. 2009. "L'entrevue semi-dirigée», dans Recherche sociale: de la problématique à la collecte de données, $5^{\mathrm{e}}$ édition, sous la dir. de B. Gauthier, Québec, Presses de l'Université du Québec, p. 337-360.

Savourey, M. 2002. Re-créer les liens familiaux, médiation familiale-soutien à la parentalité, Québec, Presses de l'Université Laval.

Sécher, R. 2010. Reconnaissance sociale et dignité des parents d'enfants placés : parentalité, précarité et protection de l'enfance, Paris, L'Harmattan.

Sellenet, C. 2000. "De la parentalité à la délégation », dans Le placement familial ou la parentalité en tensions, sous la dir. de l'Association nationale des placements familiaux, Paris, L'Harmattan, p. 57-82.

Sigurjónsdóttir, H. B. et R. Traustadóttir. 2010. «Family within a family », dans Parents with intellectual disabilities: past, present and futures, sous la dir. de G. Llewellyn, R. Traustadóttir, D. McConnell et H. B. Sigurjónsdóttir, West Sussex, John Wiley and sons Ltd., p. 49-62.

Swain, P. A. et N. Cameron. 2003. " "Good enough parenting": parental disability and child protection », Disability and Society, vol. 18, no 2, p. 165-177.

Tarleton, B. 2013. "Expanding the engagement model: the role of the specialist advocate in supporting parents with learning disabilities in child protection proceedings », Journal of Public Child Welfare, vol. 7, no 5, p. 675-690.

Traustadóttir, R. et H. B. Sigurjónsdóttir. 2010. «Parenting and resistance: strategies in dealing with services and professionals ", dans Parents with intellectual disabilities: past present and futures, sous la dir. de G. Llewellyn, R. Traustadóttir, D. McConnell et H. B. Sigurjónsdóttir, West Sussex, John Wiley and sons Ltd., p. 107-118.

Tymchuk, A. J., G. Llewellyn et M. Feldman. 1999. "Parenting by persons with intellectual disabilities: a timely international perspective », Journal on Intellectual and Developmental Disability, vol. 24, no 1, p. 3-6.

Vallerand, R. J. 2006. Les fondements de la psychologie sociale, 2e édition, Montréal, Gaëtan Morin.

Wade, C. M., R. L. Mildon et J.M. Matthews. 2007. "Service delivery to parents with an intellectual disability: family-centred or professionally centred? », Journal of Applied Research in Intellectual Disabilities, vol. 20, p. 87-98.

Walmsley, J. et J. Downer. 1997. «Shouting the loudest: self advocacy, power and diversity », dans Empowerment in everyday life : learning disability, sous la dir. de P. Ramcharan, G. Roberts, G. Grant et J. Borland, London, Jessica Kingsley Publishers, p. 35-47.

Yegidis, B. L. et R. W. Weinbach. 2006. Research Mmethods for Social Workers, 5e éd., Boston, Pearson Education. 